\title{
Affinity of Hydrated Proton at Intrinsic Water/Vapor Interface Revealed by Ion-Induced Water Alignment
}

\author{
Kuo-Yang Chiang, Laetitia Dalstein, and Yu-Chieh Wen* \\ Institute of Physics, Academia Sinica, Taipei 11529, Taiwan, R. O. C. \\ *Correspondence to: ycwen@phys.sinica.edu.tw (Y.C.W.)
}

Supporting Information for this article includes

Section S1. SFG spectra of surfaces of hydrogen halide (HX) and HX-NaX mixture solutions

Section S2. Measurement reproducibility and error propagation of the SFG analysis

Section S3. Change of the Fresnel coefficients with $\mathrm{HX}$ in water

Section S4. Amplitude of $\operatorname{Im} \chi_{S, \text { eff }}^{(2)}\left(\omega_{I R}\right)$ of the water/air interface at 3710 and $3640 \mathrm{~cm}^{-1}$ for different hydrogen halide concentrations in water

Section S5. Comparison between GC theory and modified GC theory

Section S6. Comparison between PS-SHG and PS-SFG

Section S7. Effect of discrete surface charges on the GC theory

Section S8. Effect of the adsorbed ions on the BIL spectrum

Section S9. $\chi_{B}^{(3)}\left(\omega_{I R}\right)$ spectrum of bulk water 


\section{Section S1. SFG spectra of surfaces of hydrogen halide (HX) and HX-NaX mixture solutions}
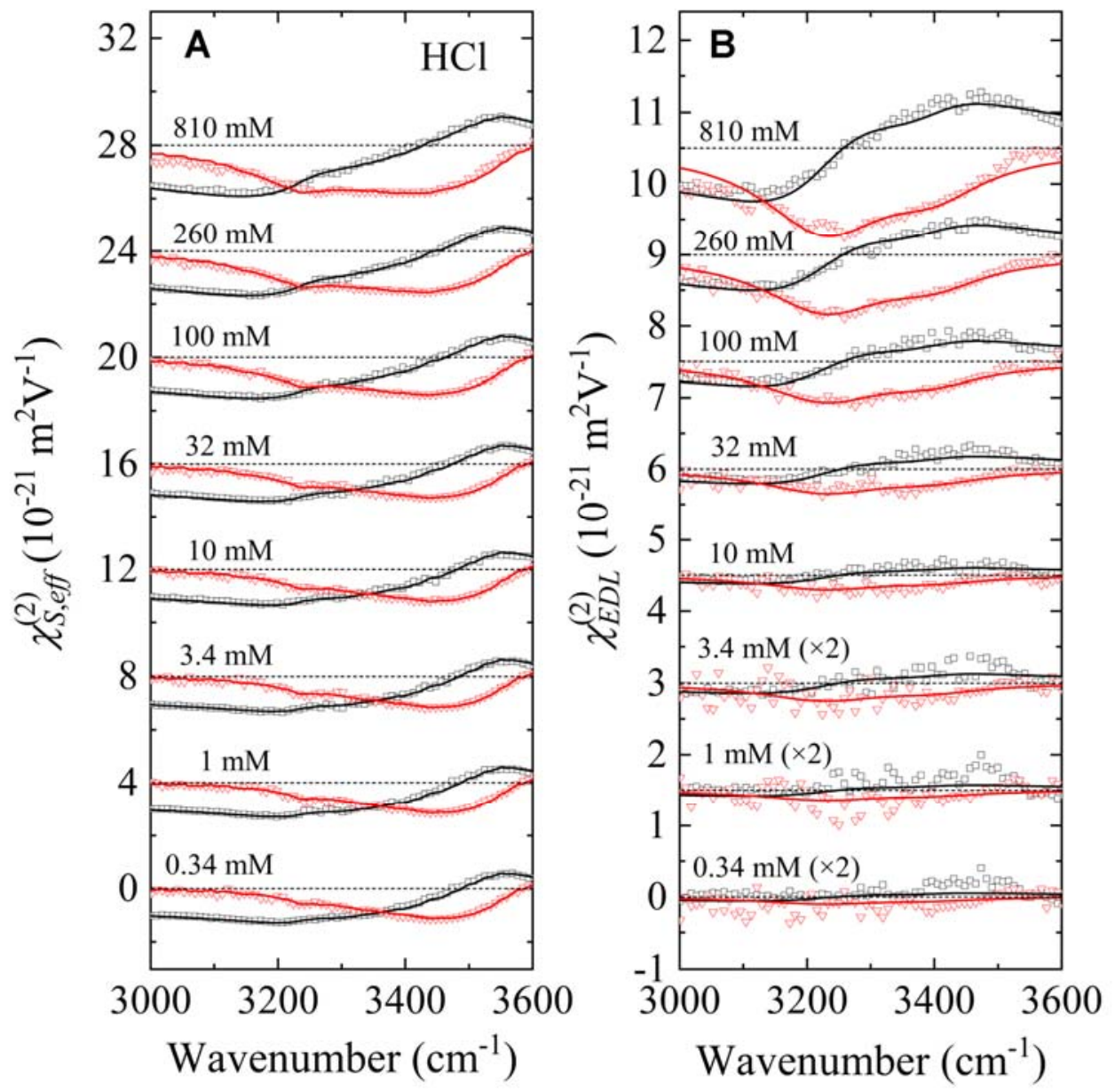

Fig. S1. (A) $\chi_{S, e f f}^{(2)}\left(\omega_{I R}\right)$ and $(B) \chi_{E D L}^{(2)}\left(\omega_{I R}\right)$ spectra of HCl-solution surfaces. Black squares and red triangles are experimental data points for real and imaginary parts of the spectra, respectively, and black and red solid curves are theoretical fits. Spectra for different $\mathrm{HCl}$ concentrations are vertically shifted for clarity. The fitting curve of $\chi_{E D L}^{(2)}\left(\omega_{I R}\right)$ is calculated from the GC theory with the reported $\chi_{B}^{(3)}\left(\omega_{I R}\right)$ of bulk water ${ }^{1}$ through Eq.(1) in the main text, and the fit of $\chi_{S, e f f}^{(2)}\left(\omega_{I R}\right)$ is obtained from the fit of $\chi_{E D L}^{(2)}\left(\omega_{I R}\right)$ plus $\chi_{B I L, 0}^{(2)}\left(\omega_{I R}\right)$ of the neat water surface. 

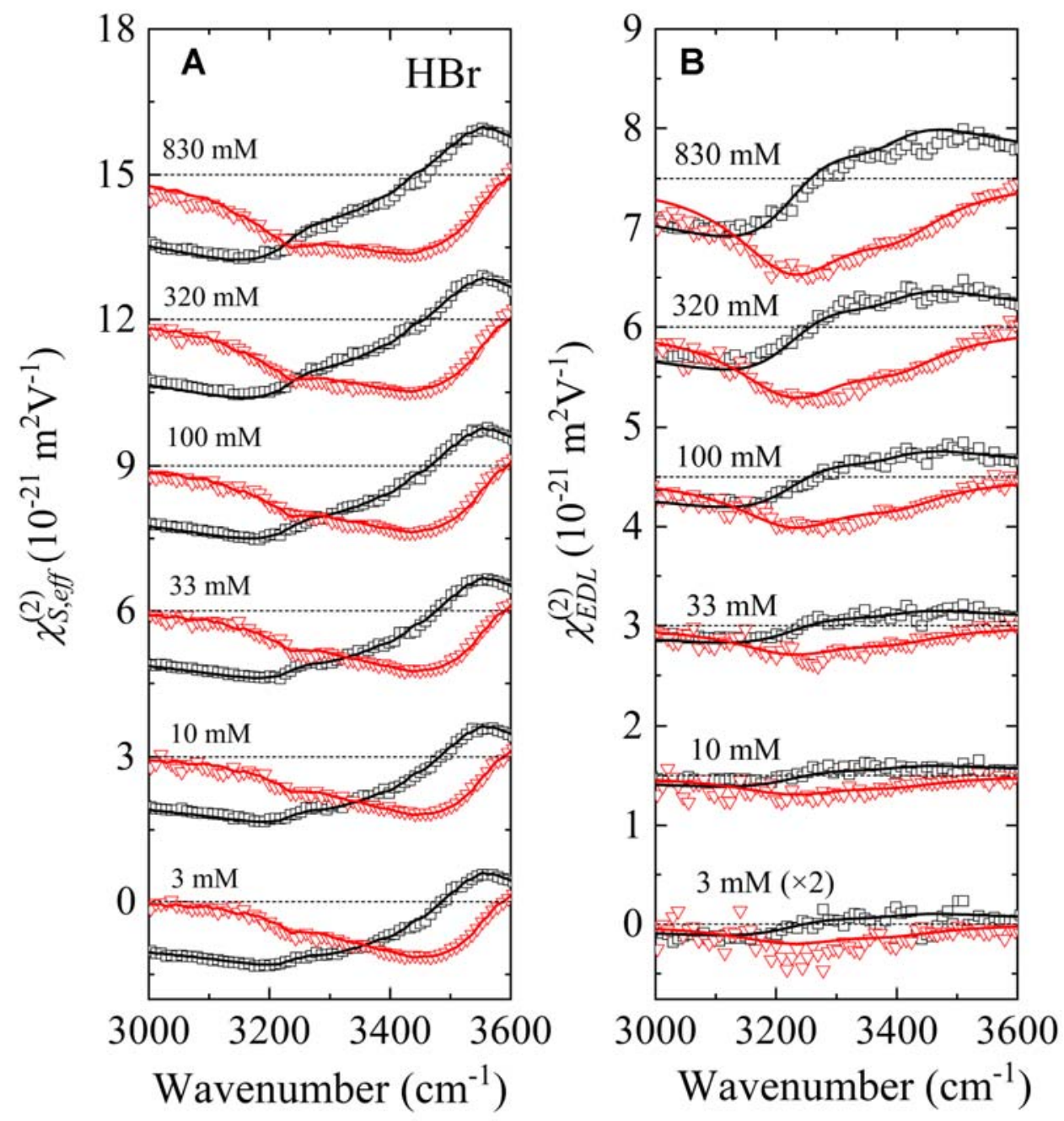

Fig. S2. (A) $\chi_{S, e f f}^{(2)}\left(\omega_{I R}\right)$ and $(B) \chi_{E D L}^{(2)}\left(\omega_{I R}\right)$ spectra of HBr-solution surfaces. Black squares and red triangles are experimental data points for real and imaginary parts of the spectra, respectively, and black and red solid curves are theoretical fits. Spectra for different $\mathrm{HBr}$ concentrations are vertically shifted for clarity. The fitting curve of $\chi_{E D L}^{(2)}\left(\omega_{I R}\right)$ is calculated from the GC theory with the reported $\chi_{B}^{(3)}\left(\omega_{I R}\right)$ of bulk water ${ }^{1}$ through Eq.(1) in the main text, and the fit of $\chi_{S, e f f}^{(2)}\left(\omega_{I R}\right)$ is obtained from the fit of $\chi_{E D L}^{(2)}\left(\omega_{I R}\right)$ plus $\chi_{B I L, 0}^{(2)}\left(\omega_{I R}\right)$ of the neat water surface. 

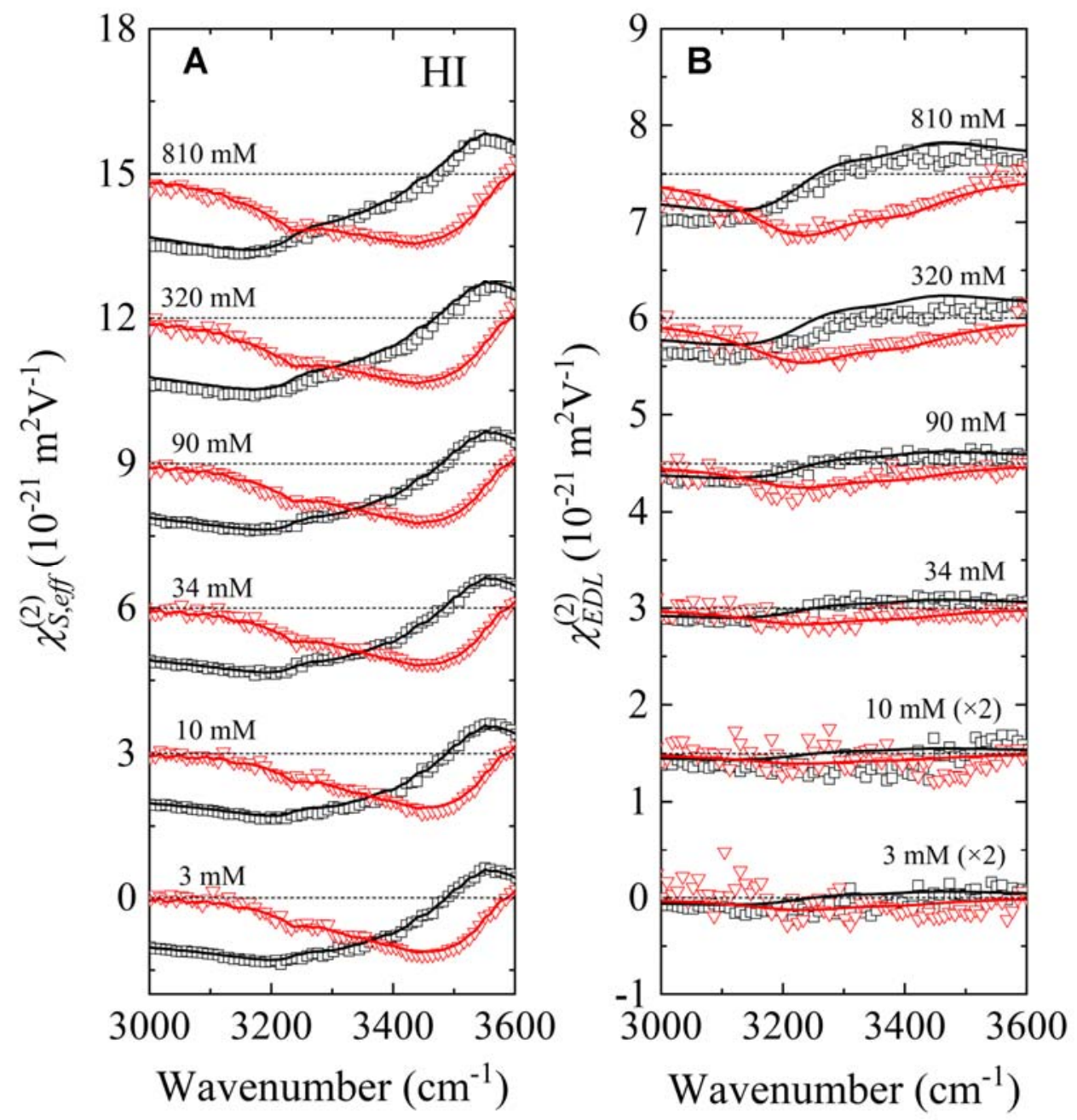

Fig. S3. (A) $\chi_{S, e f f}^{(2)}\left(\omega_{I R}\right)$ and $(B) \chi_{E D L}^{(2)}\left(\omega_{I R}\right)$ spectra of HI-solution surfaces. Black squares and red triangles are experimental data points for real and imaginary parts of the spectra, respectively, and black and red solid curves are theoretical fits. Spectra for different HI concentrations are vertically shifted for clarity. The fitting curve of $\chi_{E D L}^{(2)}\left(\omega_{I R}\right)$ is calculated from the GC theory with the reported $\chi_{B}^{(3)}\left(\omega_{I R}\right)$ of bulk water ${ }^{1}$ through Eq.(1) in the main text, and the fit of $\chi_{S, e f f}^{(2)}\left(\omega_{I R}\right)$ is obtained from the fit of $\chi_{E D L}^{(2)}\left(\omega_{I R}\right)$ plus $\chi_{B I L, 0}^{(2)}\left(\omega_{I R}\right)$ of the neat water surface.

Note that the approximation, $\left[\chi_{B I L}^{(2)}\left(\omega_{I R}\right)\right]_{\sigma} \cong \chi_{B I L, 0}^{(2)}\left(\omega_{I R}\right)$, used in obtaining $\chi_{E D L}^{(2)}\left(\omega_{I R}\right)$ from 
the measured $\chi_{S, \text { eff }}^{(2)}\left(\omega_{I R}\right)$ could become invalid for $[\mathrm{HI}]>0.2 \mathrm{M}$ (see the main text and Fig. S4 for details).
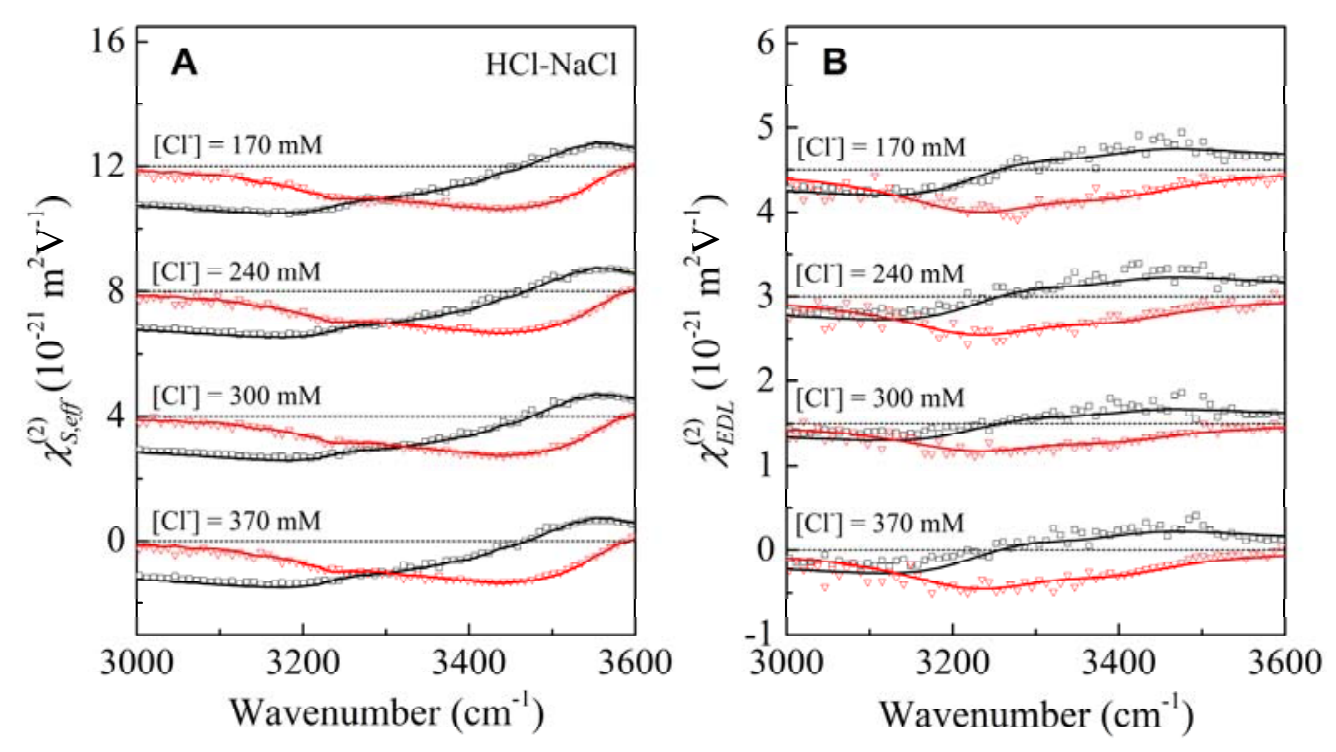

Fig. S4. (A) $\chi_{S, e f f}^{(2)}\left(\omega_{I R}\right)$ and $(B) \chi_{E D L}^{(2)}\left(\omega_{I R}\right)$ spectra of HCl-NaCl mixture solution surfaces with $\left[\mathrm{H}^{+}\right]=100 \mathrm{mM}$. Black squares and red triangles are experimental data points for real and imaginary parts of the spectra, respectively, and black and red solid curves are theoretical fits. Spectra for different $\mathrm{Cl}^{-}$concentrations are vertically shifted for clarity. The fitting curve of $\chi_{E D L}^{(2)}\left(\omega_{I R}\right)$ is calculated from the GC theory with the reported $\chi_{B}^{(3)}\left(\omega_{I R}\right)$ of bulk water ${ }^{1}$ through Eq.(1) in the main text, and the fit of $\chi_{S, e f f}^{(2)}\left(\omega_{I R}\right)$ is obtained from the fit of $\chi_{E D L}^{(2)}\left(\omega_{I R}\right)$ plus $\chi_{B I L, 0}^{(2)}\left(\omega_{I R}\right)$ of the neat water surface. 

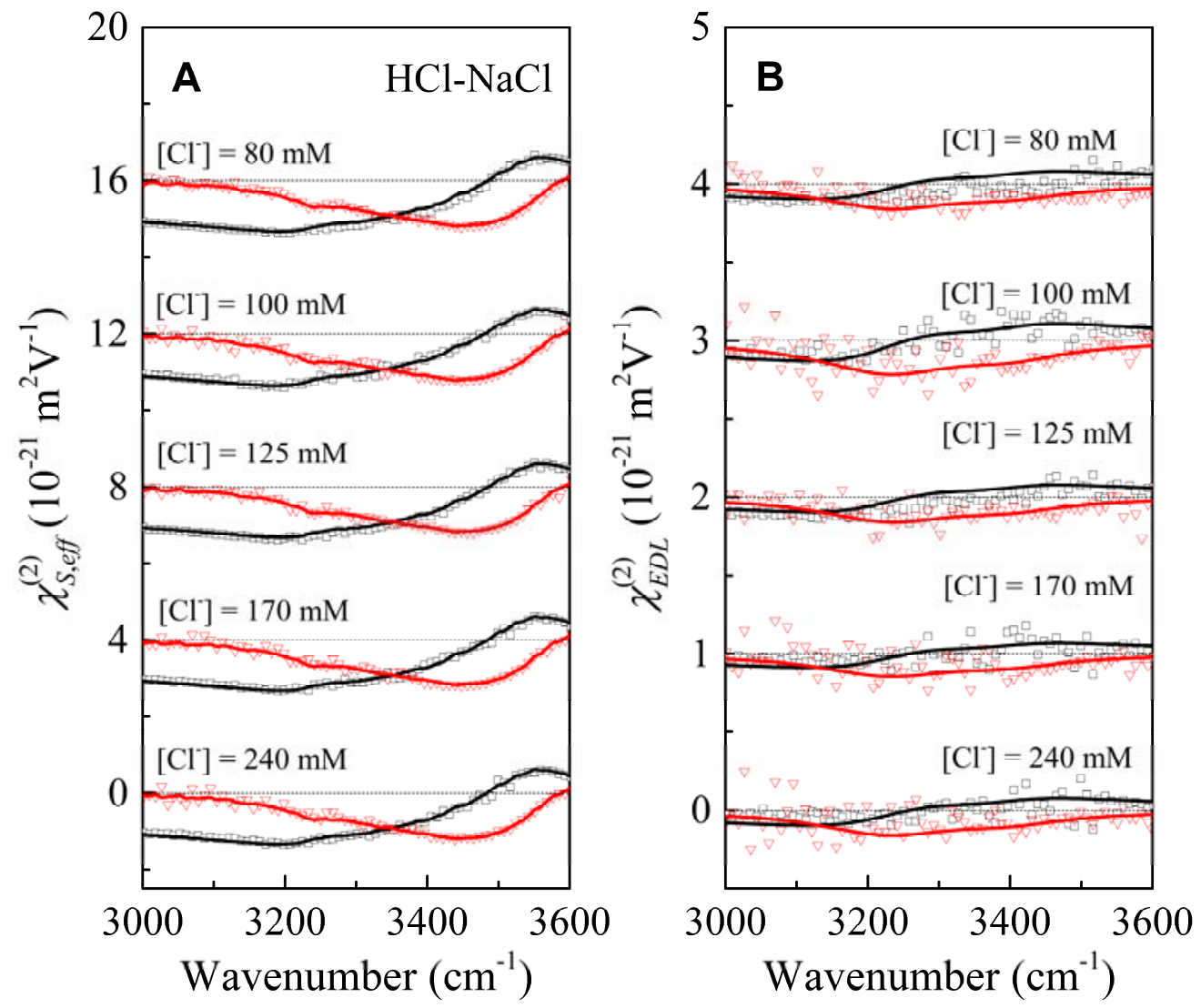

Fig. S5. (A) $\chi_{S, e f f}^{(2)}\left(\omega_{I R}\right)$ and (B) $\chi_{E D L}^{(2)}\left(\omega_{I R}\right)$ spectra of $\mathrm{HCl}-\mathrm{NaCl}$ mixture solution surfaces with $\left[\mathrm{H}^{+}\right]=33 \mathrm{mM}$. Black squares and red triangles are experimental data points for real and imaginary parts of the spectra, respectively, and black and red solid curves are theoretical fits. Spectra for different $\mathrm{Cl}^{-}$concentrations are vertically shifted for clarity. The fitting curve of $\chi_{E D L}^{(2)}\left(\omega_{I R}\right)$ is calculated from the GC theory with the reported $\chi_{B}^{(3)}\left(\omega_{I R}\right)$ of bulk water ${ }^{1}$ through Eq.(1) in the main text, and the fit of $\chi_{S, e f f}^{(2)}\left(\omega_{I R}\right)$ is obtained from the fit of $\chi_{E D L}^{(2)}\left(\omega_{I R}\right)$ plus $\chi_{B I L, 0}^{(2)}\left(\omega_{I R}\right)$ of the neat water surface. 

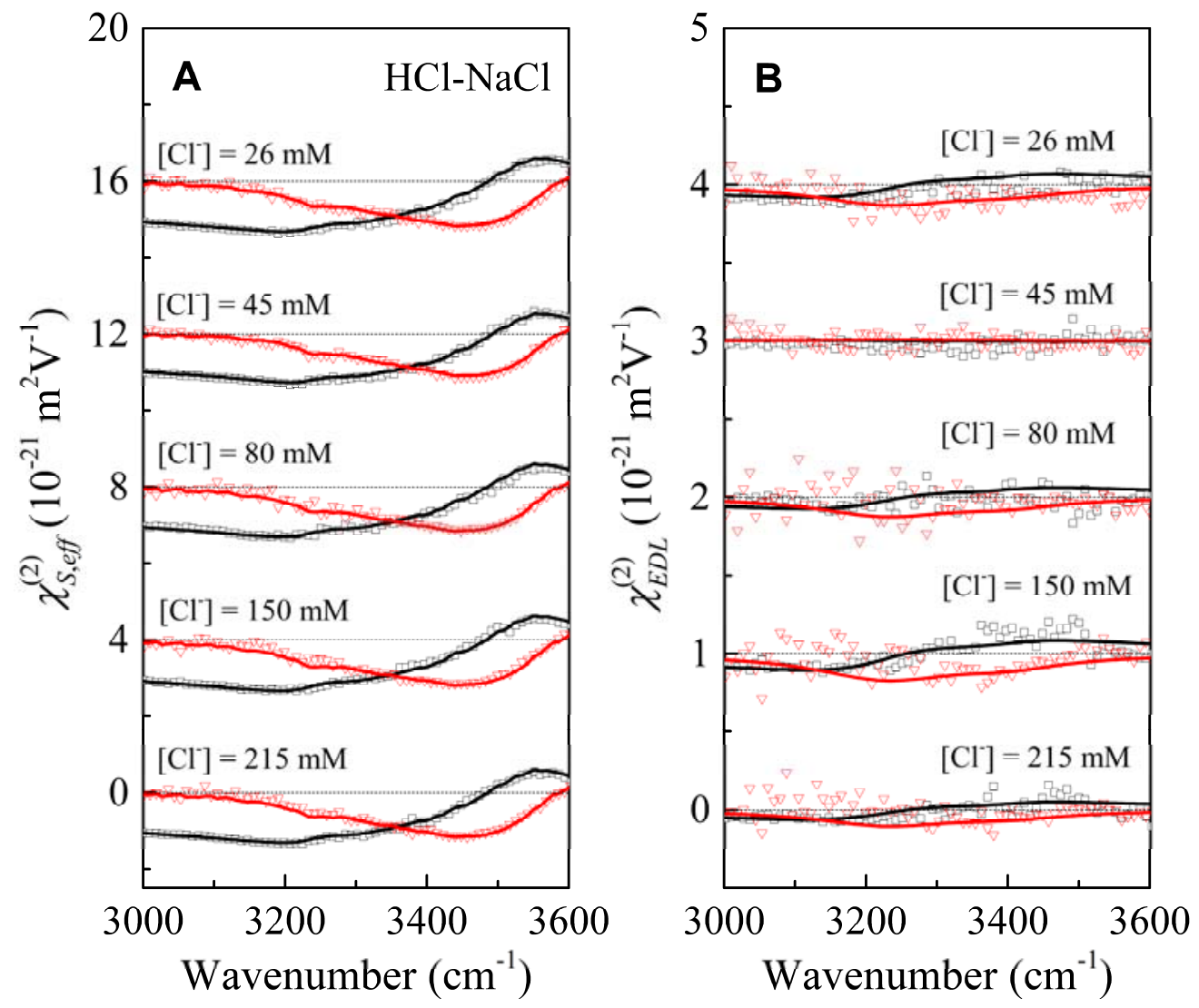

Fig. S6. $(A) \chi_{S, e f f}^{(2)}\left(\omega_{I R}\right)$ and $(B) \chi_{E D L}^{(2)}\left(\omega_{I R}\right)$ spectra of $\mathrm{HCl}-\mathrm{NaCl}$ mixture solution surfaces with $\left[\mathrm{H}^{+}\right]=10 \mathrm{mM}$. Black squares and red triangles are experimental data points for real and imaginary parts of the spectra, respectively, and black and red solid curves are theoretical fits. Spectra for different $\mathrm{Cl}^{-}$concentrations are vertically shifted for clarity. The fitting curve of $\chi_{E D L}^{(2)}\left(\omega_{I R}\right)$ is calculated from the GC theory with the reported $\chi_{B}^{(3)}\left(\omega_{I R}\right)$ of bulk water ${ }^{1}$ through Eq.(1) in the main text, and the fit of $\chi_{S, e f f}^{(2)}\left(\omega_{I R}\right)$ is obtained from the fit of $\chi_{E D L}^{(2)}\left(\omega_{I R}\right)$ plus $\chi_{B I L, 0}^{(2)}\left(\omega_{I R}\right)$ of the neat water surface. 

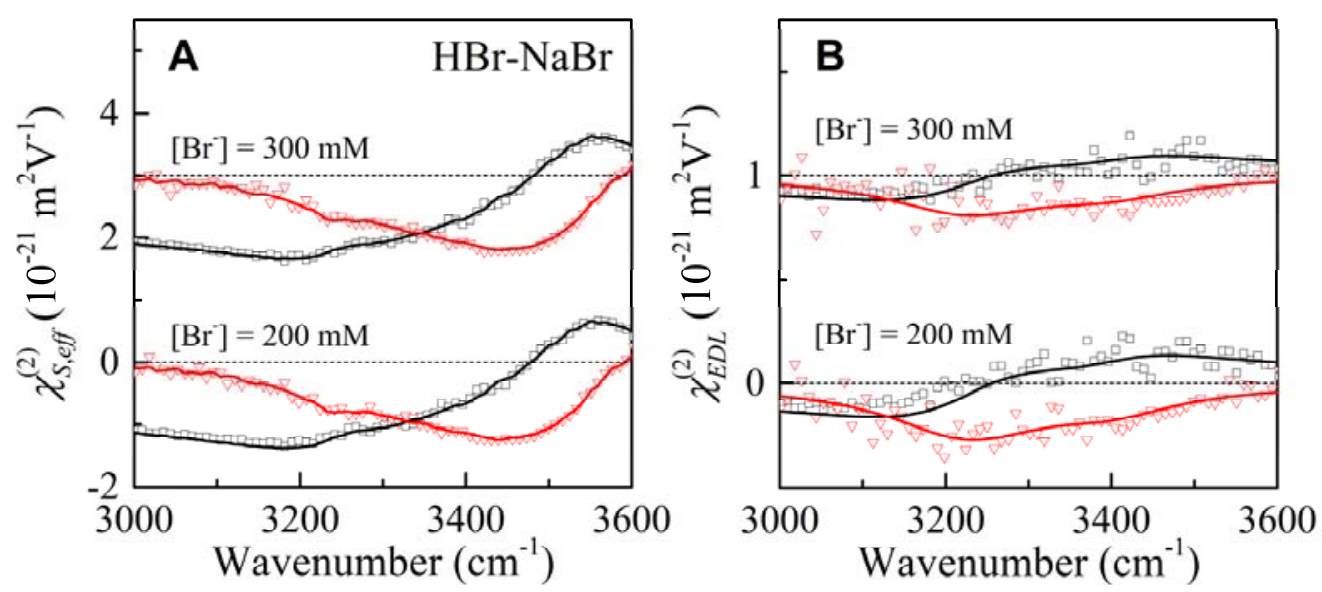

Fig. S7. (A) $\chi_{S, \text { eff }}^{(2)}\left(\omega_{I R}\right)$ and $(B) \chi_{E D L}^{(2)}\left(\omega_{I R}\right)$ spectra of $\mathrm{HBr}-\mathrm{NaBr}$ mixture solution surfaces with $\left[\mathrm{H}^{+}\right]=100 \mathrm{mM}$. Black squares and red triangles are experimental data points for real and imaginary parts of the spectra, respectively, and black and red solid curves are theoretical fits. Spectra for different $\mathrm{Br}^{-}$concentrations are vertically shifted for clarity. The fitting curve of $\chi_{E D L}^{(2)}\left(\omega_{I R}\right)$ is calculated from the GC theory with the reported $\chi_{B}^{(3)}\left(\omega_{I R}\right)$ of bulk water ${ }^{1}$ through Eq.(1) in the main text, and the fit of $\chi_{S, e f f}^{(2)}\left(\omega_{I R}\right)$ is obtained from the fit of $\chi_{E D L}^{(2)}\left(\omega_{I R}\right)$ plus $\chi_{B I L, 0}^{(2)}\left(\omega_{I R}\right)$ of the neat water surface. 

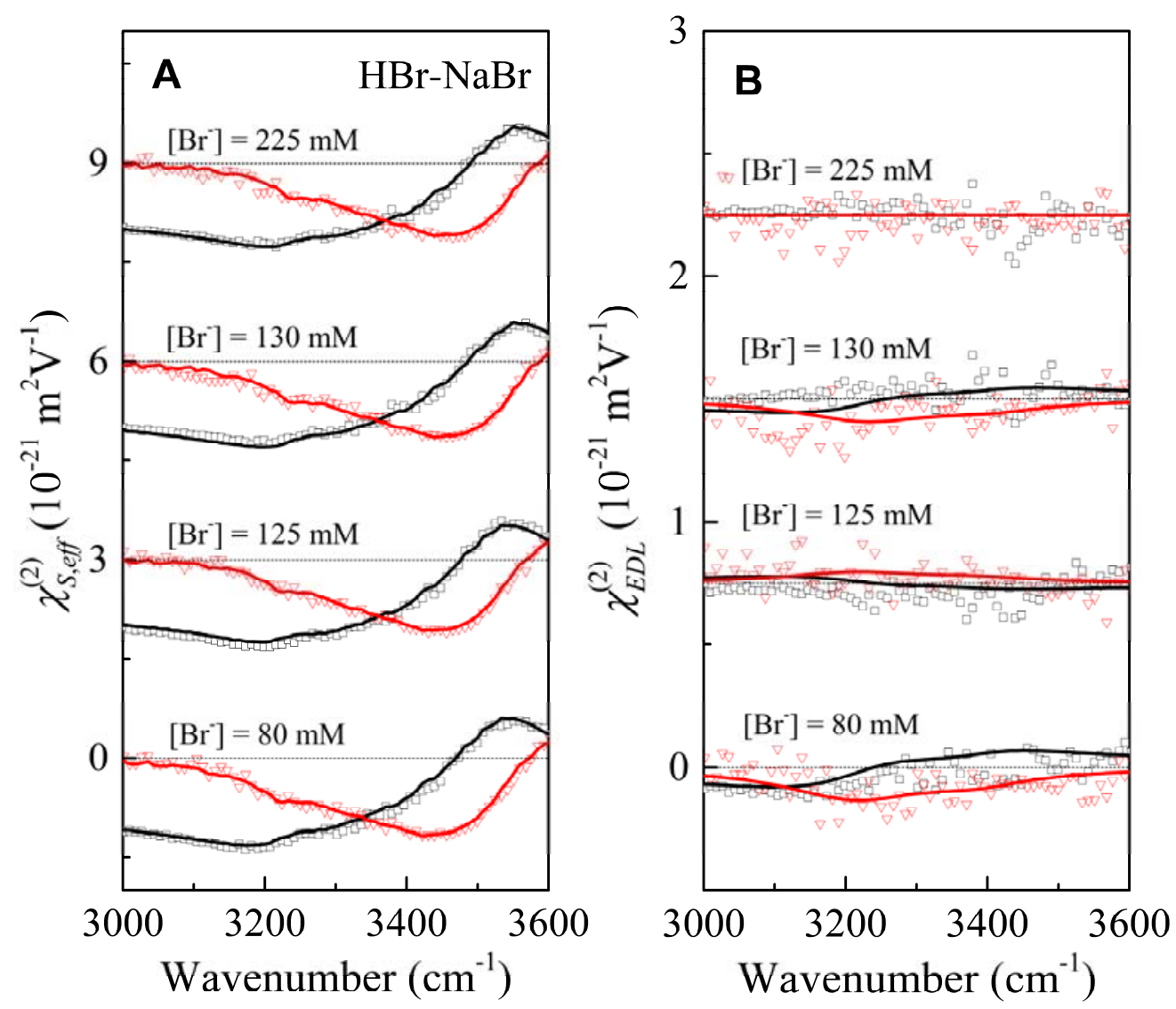

Fig. S8. $(A) \chi_{S, e f f}^{(2)}\left(\omega_{I R}\right)$ and $(B) \chi_{E D L}^{(2)}\left(\omega_{I R}\right)$ spectra of $\mathrm{HBr}-\mathrm{NaBr}$ mixture solution surfaces with $\left[\mathrm{H}^{+}\right]=33 \mathrm{mM}$. Black squares and red triangles are experimental data points for real and imaginary parts of the spectra, respectively, and black and red solid curves are theoretical fits. Spectra for different $\mathrm{Br}^{-}$concentrations are vertically shifted for clarity. The fitting curve of $\chi_{E D L}^{(2)}\left(\omega_{I R}\right)$ is calculated from the GC theory with the reported $\chi_{B}^{(3)}\left(\omega_{I R}\right)$ of bulk water ${ }^{1}$ through Eq.(1) in the main text, and the fit of $\chi_{S, e f f}^{(2)}\left(\omega_{I R}\right)$ is obtained from the fit of $\chi_{E D L}^{(2)}\left(\omega_{I R}\right)$ plus $\chi_{B I L, 0}^{(2)}\left(\omega_{I R}\right)$ of the neat water surface. 

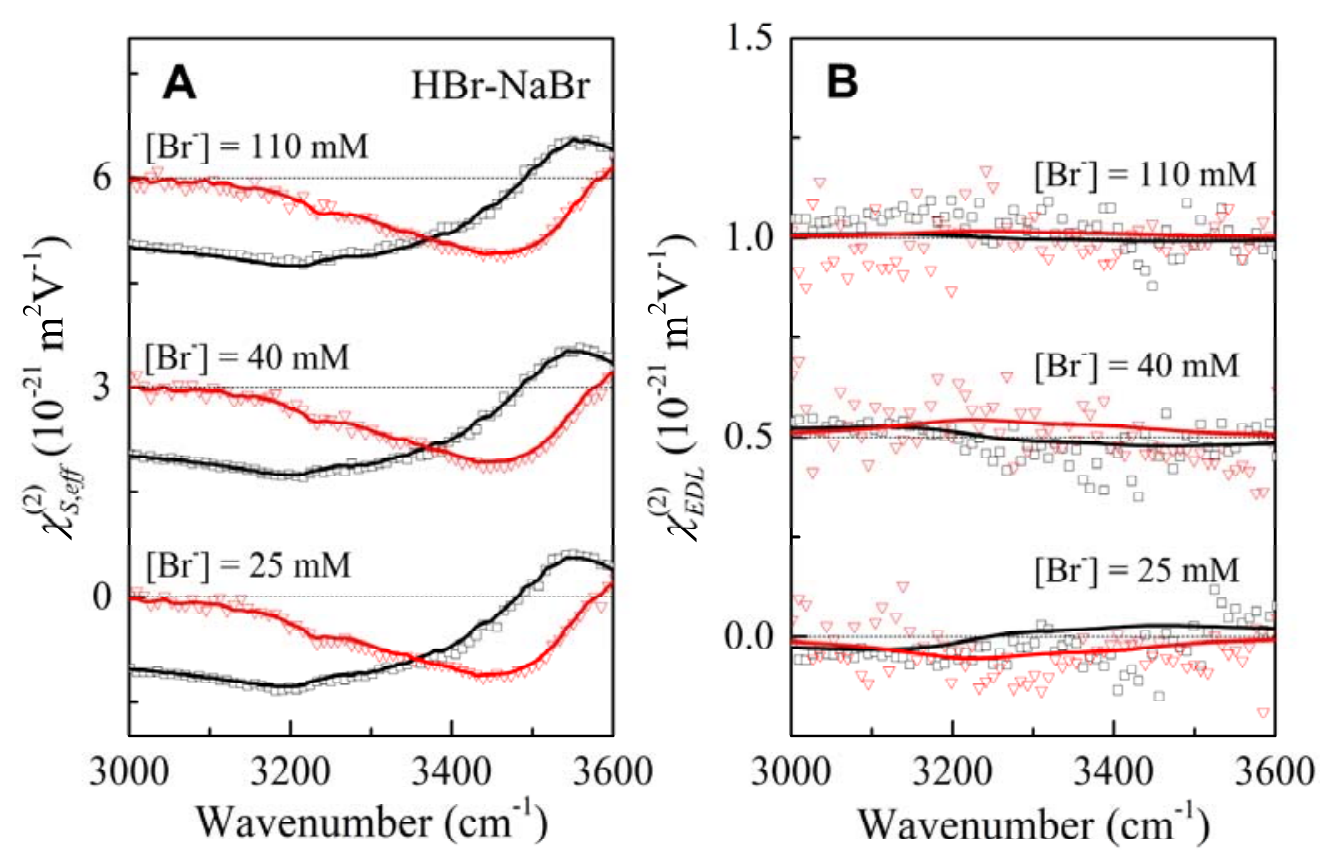

Fig. S9. (A) $\chi_{S, \text { eff }}^{(2)}\left(\omega_{I R}\right)$ and $(B) \chi_{E D L}^{(2)}\left(\omega_{I R}\right)$ spectra of $\mathrm{HBr}-\mathrm{NaBr}$ mixture solution surfaces with $\left[\mathrm{H}^{+}\right]=10 \mathrm{mM}$. Black squares and red triangles are experimental data points for real and imaginary parts of the spectra, respectively, and black and red solid curves are theoretical fits. Spectra for different $\mathrm{Br}^{-}$concentrations are vertically shifted for clarity. The fitting curve of $\chi_{E D L}^{(2)}\left(\omega_{I R}\right)$ is calculated from the GC theory with the reported $\chi_{B}^{(3)}\left(\omega_{I R}\right)$ of bulk water ${ }^{1}$ through Eq.(1) in the main text, and the fit of $\chi_{S, e f f}^{(2)}\left(\omega_{I R}\right)$ is obtained from the fit of $\chi_{E D L}^{(2)}\left(\omega_{I R}\right)$ plus $\chi_{B I L, 0}^{(2)}\left(\omega_{I R}\right)$ of the neat water surface. 

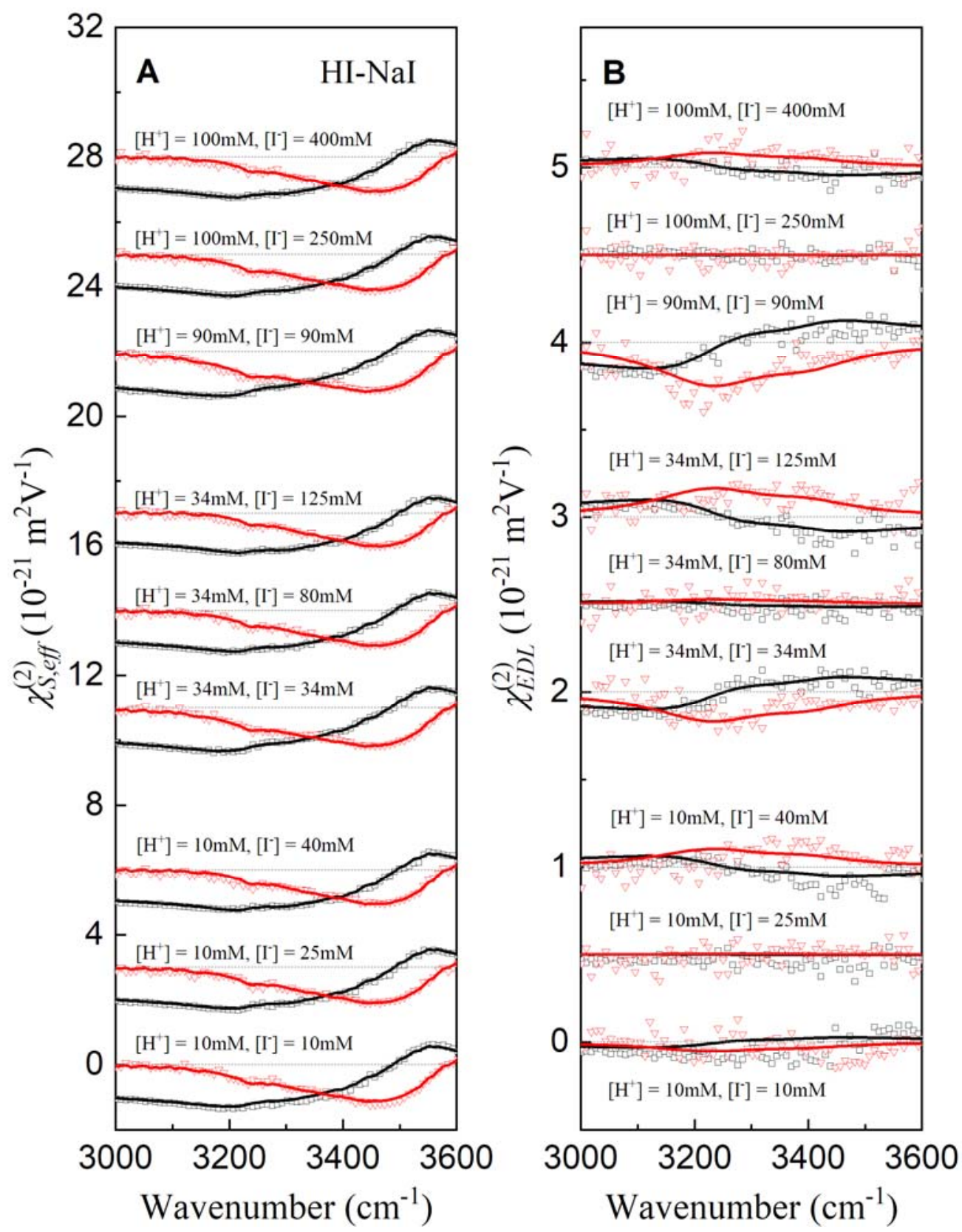

Fig. S10. (A) $\chi_{S, e f f}^{(2)}\left(\omega_{I R}\right)$ and $(B) \chi_{E D L}^{(2)}\left(\omega_{I R}\right)$ spectra of HI-NaI mixture solution surfaces for different $\left[\mathrm{H}^{+}\right]$and $\left[\mathrm{I}^{-}\right]$. Spectra of pure HI solutions in Fig. S3 are also shown for comparison. Black squares and red triangles are experimental data points for real and imaginary parts of the spectra, respectively, and black and red solid curves are theoretical fits. Spectra are vertically shifted for clarity. The fitting curve of $\chi_{E D L}^{(2)}\left(\omega_{I R}\right)$ is calculated from the GC theory with the 
reported $\chi_{B}^{(3)}\left(\omega_{I R}\right)$ of bulk water ${ }^{1}$ through Eq.(1) in the main text, and the fit of $\chi_{S, \text { eff }}^{(2)}\left(\omega_{I R}\right)$ is obtained from the fit of $\chi_{E D L}^{(2)}\left(\omega_{I R}\right)$ plus $\chi_{B I L, 0}^{(2)}\left(\omega_{I R}\right)$ of the neat water surface.

\section{Section S2. Measurement reproducibility and error propagation of the SFG analysis}

To evaluate reproducibility and noise level of our experiments, we repeated PS-SFVS measurements of the neat water/vapor surface for 17 separate samples in different days. Fig. S11 shows all the measured $\chi_{S, e f f}^{(2)}\left(\omega_{I R}\right)$ spectra and the standard deviation between the spectra in the bonded-OH range. It is seen that the standard deviation for both $\operatorname{Re} \chi_{S, \text { eff }}^{(2)}\left(\omega_{I R}\right)$ and $\operatorname{Im} \chi_{S, \text { eff }}^{(2)}\left(\omega_{I R}\right)$ is only about $3 \sim 10 \times 10^{-23} \mathrm{~m}^{2} / \mathrm{V}$, corresponding to $3 \sim 9 \%$ of the strength of the bonded $\mathrm{OH}$ band in the $\operatorname{Im} \chi_{S, \text { eff }}^{(2)}\left(\omega_{I R}\right)$ spectrum $\left(\sim 1.1 \times 10^{-21} \mathrm{~m}^{2} / \mathrm{V}\right.$ at $\left.\sim 3450 \mathrm{~cm}^{-1}\right)$. The good data correlation supports high reproducibility and signal-to-noise ratio of our measurements. Note that the data correlation is also revealed by monotonic variations of the measured $\chi_{S, \text { eff }}^{(2)}\left(\omega_{I R}\right)$ spectra when altering $\mathrm{HX}$ or $\mathrm{NaX}$ concentrations in water (49 spectra in Fig. S1-S10).
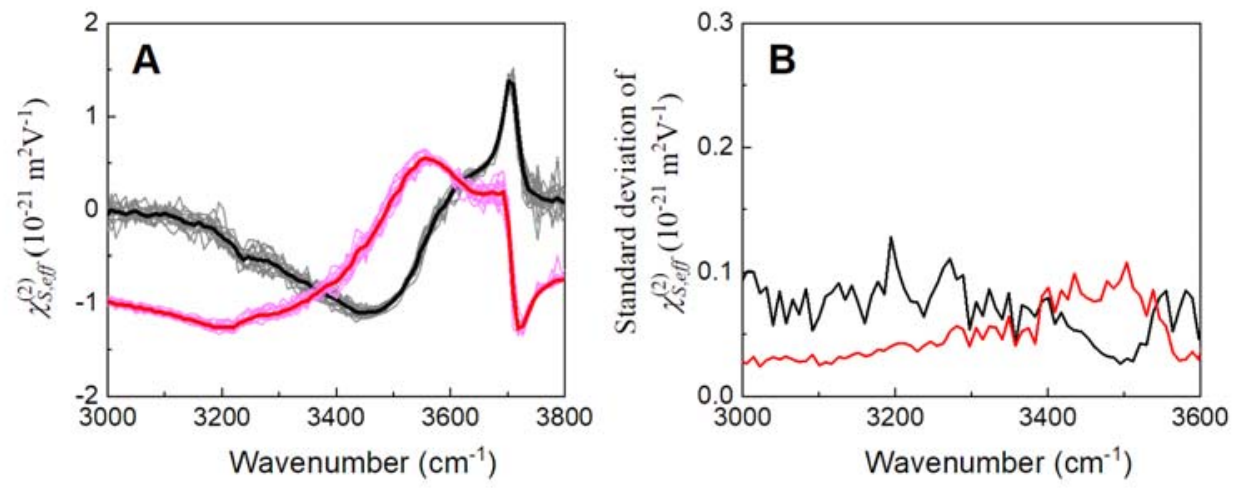

Fig. S11 $(A) \chi_{S, e f f}^{(2)}\left(\omega_{I R}\right)$ spectra of the neat water surface measured from 17 separate samples in different days (thin lines) and their average spectra (thick lines). (B) Standard deviation between 
the $17 \chi_{S, \text { eff }}^{(2)}\left(\omega_{I R}\right)$ spectra in $A$ as a function of IR frequency in the bonded-OH range. In $A$ and $B$, red and black curves are real and imaginary parts of the spectra, respectively. Distinct from recent observations by Sun and $\operatorname{Tian}^{2}$, our measured $\operatorname{Im} \chi_{S, e f f}^{(2)}\left(\omega_{I R}\right)$ does not reveal discernable positive offset at low frequencies $\left(\sim 3000 \mathrm{~cm}^{-1}\right)$.

In analyzing $\sigma$ for a given acidic solution, we first averaged the 17 neat water spectra in Fig. S11 to obtain a high-quality reference $\chi_{B I L, 0}^{(2)}\left(\omega_{I R}\right)$ spectrum, and then extracted $\chi_{E D L}^{(2)}\left(\omega_{I R}\right)$ of the acidic solution from the measured $\chi_{S, \text { eff }}^{(2)}\left(\omega_{I R}\right)$ through $\chi_{E D L}^{(2)}\left(\omega_{I R}\right) \cong \chi_{S, \text { eff }}^{(2)}\left(\omega_{I R}\right)-$ $\chi_{B I L, 0}^{(2)}\left(\omega_{I R}\right)$ (see the main text for details). Subsequently, we adopted two mathematically equivalent approaches to estimate $\sigma$ from $\chi_{E D L}^{(2)}\left(\omega_{I R}\right)$. First, we used Eq.(1) in the main text and $E_{0}(z)$ to fit the measured $\chi_{E D L}^{(2)}\left(\omega_{I R}\right)$ spectrum with the known $\chi_{B}^{(3)}\left(\omega_{I R}\right)$ spectrum $^{1}$ and $\Delta k_{z}$, where $E_{0}(z)$ was calculated from the GC theory with the fitting variable $\sigma$. (See the main text for details.) Second, we deduced complex $\Psi$ directly from the measured $\chi_{E D L}^{(2)}\left(\omega_{I R}\right)$ via $\Psi\left(\omega_{I R}\right)=$ $\chi_{E D L}^{(2)}\left(\omega_{I R}\right) / \chi_{B}^{(3)}\left(\omega_{I R}\right)$ with the known $\chi_{B}^{(3)}\left(\omega_{I R}\right)$ spectrum ${ }^{1}$, and then obtained $\sigma$ from $\Psi$ through the GC theory and Eq.(1) in the main text with the known $\Delta k_{z}$. Although the two approaches are mathematically equivalent, the second approach was adopted to follow the error propagation from the measured spectra to $\sigma$ with unambiguous statistical definition. Here we use $10 \mathrm{mM}-\mathrm{HCl}$ solution as an example for explaining the error propagation. Fig. S12 shows the complex $\Psi\left(\omega_{I R}\right)$ deduced from $\chi_{E D L}^{(2)}\left(\omega_{I R}\right)$ of the $10 \mathrm{mM}-\mathrm{HCl}$ solution (see Fig. S1 for spectra). It is seen that the deduced $\Psi$ is independent of the IR frequency within experimental error, in agreement with the expectation for $\Psi$ being a characteristic of the surface field distribution. 
Statistical analysis of $\Psi\left(\omega_{I R}\right)$ over the bonded-OH spectral region $\left(3100-3500 \mathrm{~cm}^{-1}\right)$ yields $|\Psi|$ $=5.7 \pm 2.2 \mathrm{mV}$ and phase of $\Psi$ of $12 \pm 30$ degrees, where the uncertainties are determined as the standard deviation. Note that with low $|\Psi|$ for most of the samples, we cannot determine the phase of $\Psi$ with meaningful precision, so that we relied only on the absolute value of $\Psi$ (and its uncertainty) to estimate the mean and the standard deviation of $\sigma$. For the $10 \mathrm{mM} \mathrm{HCl}$ solution with $|\Psi|=5.7 \pm 2.2 \mathrm{mV}$, we get $\sigma /|e|=(8.8 \pm 3.2) \times 10^{-3} \mathrm{~nm}^{-2}$, which is near the detection limit of our instrument.

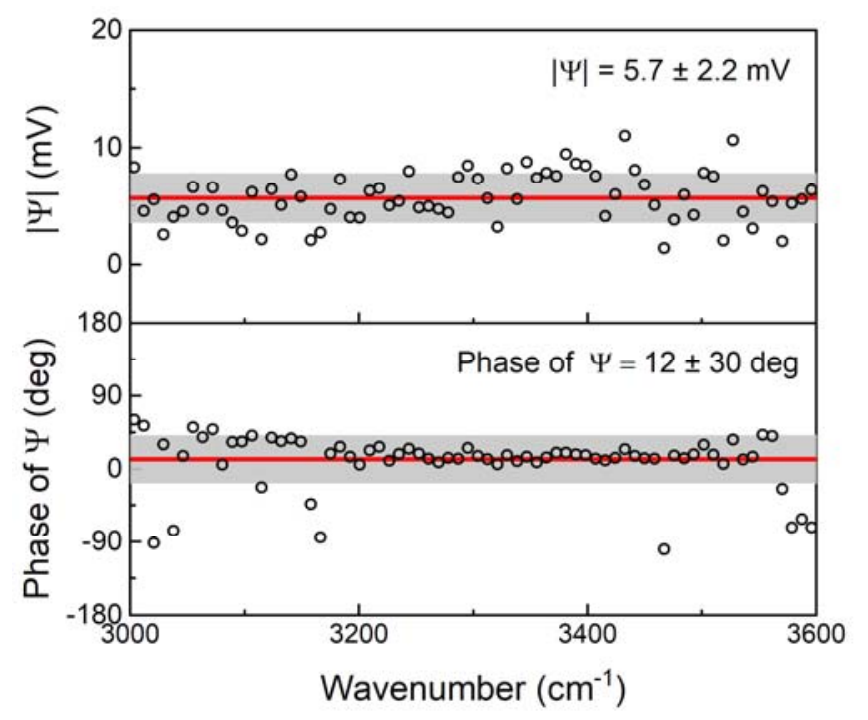

Fig. S12 Amplitude and phase of $\Psi\left(\omega_{I R}\right)$ deduced from the measured $\chi_{S, \text { eff }}^{(2)}\left(\omega_{I R}\right)$ spectrum of the 10-mM HCl solution surface in Fig. S1 (dot). Average and standard deviation of the measured $\Psi\left(\omega_{I R}\right)$ are indicated and plotted by horizontal red lines with shadowed regions.

Here we describe the error propagation from $\sigma$ to the subsequently deduced properties of ion adsorption in order. First, as described in the main text, the slope of $\sigma$ for the HX-NaX mixture solution versus $\left[\mathrm{X}^{-}\right]$in Fig. 4 is associated with $K\left(\mathrm{X}^{-}\right)$of halide ions. To follow the error 
propagation from $\sigma$ to $K\left(\mathrm{X}^{-}\right)$, we fitted the data in Fig. 4 for each halide species with different $\left[\mathrm{X}^{-}\right]$and $\left[\mathrm{H}^{+}\right]$by straight lines with a single constant slope, and the slope was tuned as a fitting variable to cover the entire data set of $\sigma$ including their error bars, yielding the uncertainty range of the slope and so for $K\left(\mathrm{X}^{-}\right)$. Second, for a HX solution, we calculated $\rho\left(\mathrm{X}^{-}\right)$from $K\left(\mathrm{X}^{-}\right)$ with the given $\left[\mathrm{X}^{-}\right]$, and then extracted $\rho\left(\mathrm{H}_{3} \mathrm{O}^{+}\right)$from the measured $\sigma$ with the estimated $\rho\left(\mathrm{X}^{-}\right)$(see the main text for details). Here, the uncertainty of $K\left(\mathrm{X}^{-}\right)$induced errors of the estimated $\rho\left(\mathrm{X}^{-}\right)$, which, together with uncertainty of the measured $\sigma$ of the solution, were transferred to the error of the deduced $\rho\left(\mathrm{H}_{3} \mathrm{O}^{+}\right)$, as illustrated in Fig. S13. Third, in Fig. 3B in the main text, we fitted $\rho\left(\mathrm{H}_{3} \mathrm{O}^{+}\right)$of different hydrogen halide solutions with varying [HX] by $\rho\left(\mathrm{H}_{3} \mathrm{O}^{+}\right)=K\left(\mathrm{H}_{3} \mathrm{O}^{+}\right) \cdot\left[\mathrm{H}^{+}\right]$for evaluating $K\left(\mathrm{H}_{3} \mathrm{O}^{+}\right)$. Uncertainty of the deduced $K\left(\mathrm{H}_{3} \mathrm{O}^{+}\right)$ was affected by the error propagation from $\rho\left(\mathrm{H}_{3} \mathrm{O}^{+}\right)$and found to be similar with that of $K\left(\mathrm{X}^{-}\right)$, which was about $1 \AA$. Finally, we calculated $\mathrm{Kv}_{\mathrm{v}}$ of $\mathrm{H}_{3} \mathrm{O}^{+}$from $K$ via $K \mathrm{~V} \approx K / L$. Note that $L$ taken from theoretical reports has considerable uncertainties (more than $50 \%$ ), which, together with the error of $K$, propagated through $K_{\mathrm{V}}$ to $\Delta \mathrm{G}\left[=k_{B} T \cdot \ln \left(K_{V}\right)\right]$ and $\Delta \mathrm{pH}\left[=-\log \left(K_{V}\right)\right]$, where $k_{B} T$ is the thermal energy at room temperature.
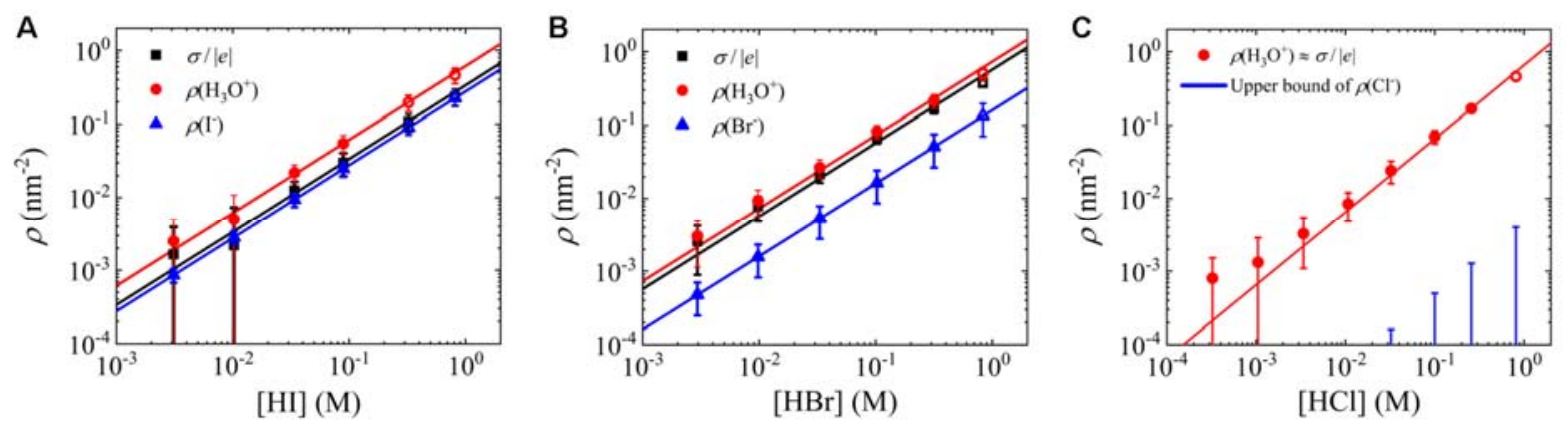

Fig. S13. Net surface charge density $\sigma$ and surface ion density $\rho$ at surfaces of $(A) \mathrm{HI},(B) \mathrm{HBr}$, and $(C) \mathrm{HCl}$ solutions with various concentrations. Black squares are $\sigma /|e|$ deduced from the 
measured $\chi_{E D L}^{(2)}\left(\omega_{I R}\right)$, which equal to the difference of the surface density between cations and anions, i.e., $\sigma /|e|=\rho\left(\mathrm{H}_{3} \mathrm{O}^{+}\right)-\rho\left(\mathrm{X}^{-}\right)$. Blue triangles are $\rho\left(\mathrm{X}^{-}\right)$estimated from the measured $K\left(\mathrm{X}^{-}\right)$with the given $\left[\mathrm{X}^{-}\right]$, and the red circles are $\rho\left(\mathrm{H}_{3} \mathrm{O}^{+}\right)$obtained from the difference between the measured $\sigma /|e|$ and the estimated $\rho\left(\mathrm{X}^{-}\right)$. [For $\mathrm{HCl}$ solutions, $K\left(\mathrm{Cl}^{-}\right)$is essentially small so that we can only estimate the upper bound of $\rho\left(\mathrm{Cl}^{-}\right)$and have $\sigma /|e| \approx$ $\rho\left(\mathrm{H}_{3} \mathrm{O}^{+}\right)$.] We depict the dots by solid (open) format for denoting that the approximations used in analysis of the data point are (not) fully justified. (See the main text for details.) Lines are fitting curves assuming a constant ratio between $\sigma /|e|$ (or $\rho$ ) and [HX].

\section{Section S3. Change of the Fresnel coefficients with $\mathrm{HX}$ in water}

We examine the effect of ions in water on the Fresnel coefficient $L_{i i}$ and its consequence on our spectral analysis. Expressions of $L_{i i}$ and the interfacial dielectric constant can be found elsewhere ${ }^{3}$, and the frequency-dependent complex refractive index of the solutions was taken into account in the calculation of $\mathrm{Lii}^{4-5}$ Shown in Fig. S14 are the calculated amplitude and phase of the product of the three Fresnel coefficients associated with our SSP-polarized SFG measurement. The result for the neat water surface is the same as that reported earlier ${ }^{2}$ and compared here with a 2-M HCl solution, as a representative case of the strongest ion effect in our study. It is found that the maximum deviation between the two products of the Fresnel coefficients with IR in the OHstretch range is only $\sim 2 \%$ in amplitude and $\sim 1$ degree in phase. Such changes are essentially within our experimental uncertainty (see SI section 2). We can, therefore, approximate the Fresnel coefficients for all the solutions studied as that of the neat water surface in the spectral analysis. 
Also notice that the weak ion effect on Fresnel coefficients was recently reported by Urashima et. al. for the silica/ $\mathrm{NaCl}$-solution interface. ${ }^{6}$

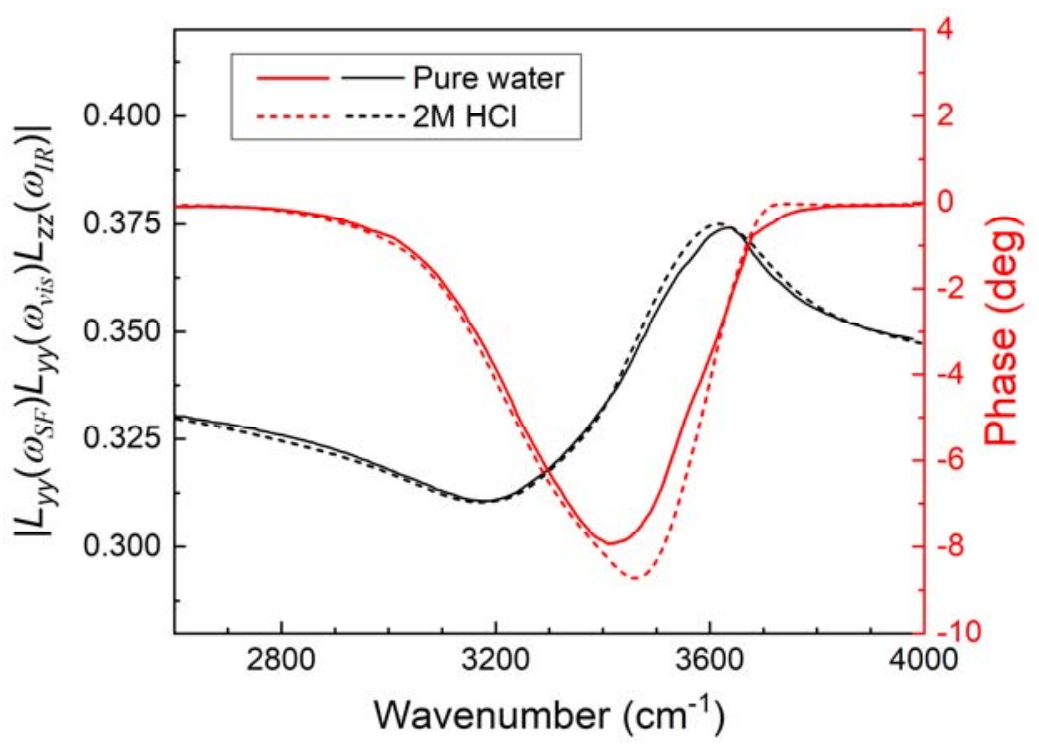

Fig. S14. Amplitude and phase of the product of the Fresnel coefficients, $L_{y y}\left(\omega_{S F}\right) L_{y y}\left(\omega_{v i s}\right) L_{z z}\left(\omega_{I R}\right)$, for surfaces of the neat water and $2 \mathrm{M} \mathrm{HCl}$ solution. The subscript of $L_{i i}$ refers to particular tensorial Fresnel factor with $z$ defined along the surface normal and the optical plane set on the $x$-z plane. (See SI of Ref. ${ }^{3}$ for detailed expression of $L_{i i}$ ) 
Section S4. Amplitude of $\operatorname{Im} \chi_{S, e f f}^{(2)}\left(\omega_{I R}\right)$ of the water/air interface at 3710 and $3640 \mathrm{~cm}^{-1}$ for different hydrogen halide concentrations in water
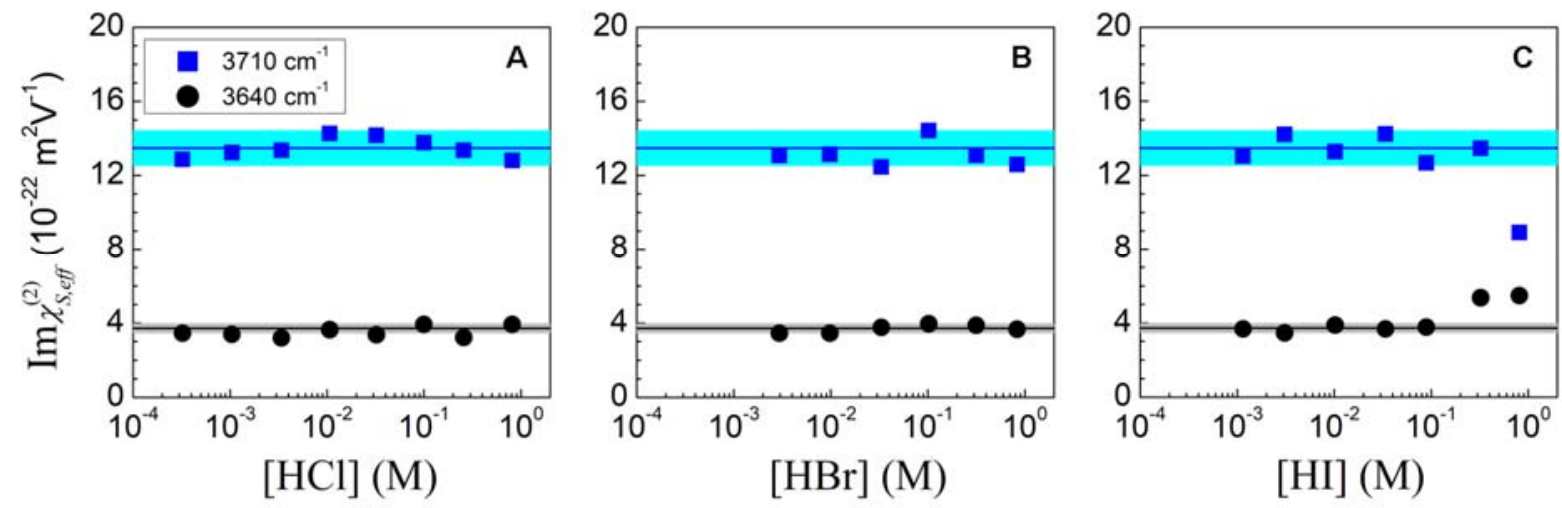

Fig. S15. $\operatorname{Im} \chi_{S, \text { eff }}^{(2)}\left(\omega_{I R}\right)$ of the water/air interface at 3710 and $3640 \mathrm{~cm}^{-1}$ for different $(A) \mathrm{HCl},(B)$ $\mathrm{HBr}$, and (C) $\mathrm{HI}$ concentrations in water (dots). $3710 \mathrm{~cm}^{-1}$ and $3640 \mathrm{~cm}^{-1}$ correspond to the amplitudes of the dangling $\mathrm{OH}$ peak and the surrounding positive hump in the $\operatorname{Im} \chi_{S, \text { eff }}^{(2)}\left(\omega_{I R}\right)$ spectrum, respectively (see Fig. 1 in the main text for the spectra). Lines and shadow regions denote the average and the uncertainty range of $\operatorname{Im} \chi_{S, e f f}^{(2)}\left(\omega_{I R}\right)$ obtained from 17 measurements of the neat water/vapor interface. Except the data points with 320 and $810 \mathrm{mM} \mathrm{HI}$, the results from the hydrogen halide solutions and the neat water are found to be consistent within our measurement error, suggesting a constant structure of the BIL. 


\section{Section S5. Comparison between GC theory and modified GC theory}

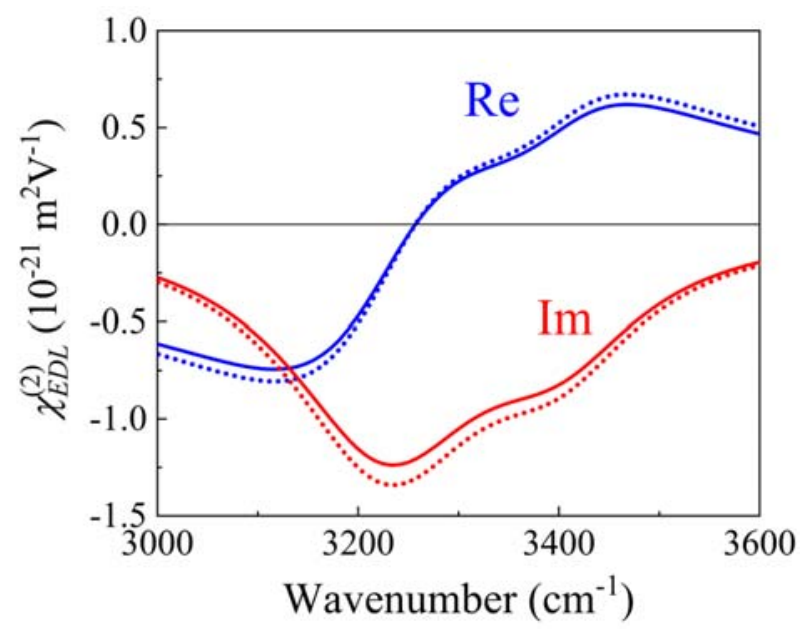

Fig. S16. Complex $\chi_{E D L}^{(2)}\left(\omega_{I R}\right)$ spectra calculated from the GC theory (solid lines) and the modified GC theory (dotted lines) for the surface of the $0.8 \mathrm{M} \mathrm{HCl}$ solution. Here we aim to clarify whether our spectral analysis is under influence of the steric effect of ions at high ion concentrations. This test was performed by comparing the calculations using the GC theory with that based on a modified GC theory ${ }^{7}$ that takes the finite-size effect of the ions into account. [In using the modified GC theory, we follow Ref. ${ }^{7-8}$ to assume the effective radius of the ions as the Bjerrum length in water at room temperature $(0.7 \mathrm{~nm})$.] We consider the surface of $0.8 \mathrm{M} \mathrm{HCl}$ solution which has the highest $\sigma$ in the presented study. The solid lines based on the GC theory with a particular value of $\sigma$ are the fitting curves for the measured complex $\chi_{E D L}^{(2)}\left(\omega_{I R}\right)$. The dotted lines are calculated from the modified GC theory with the same value of $\sigma$. The two calculation results are essentially similar within our experimental errors, indicating an insignificant steric effect of ions on our spectral analysis for $\sigma$. 


\section{Section S6. Comparison between PS-SHG and PS-SFG}

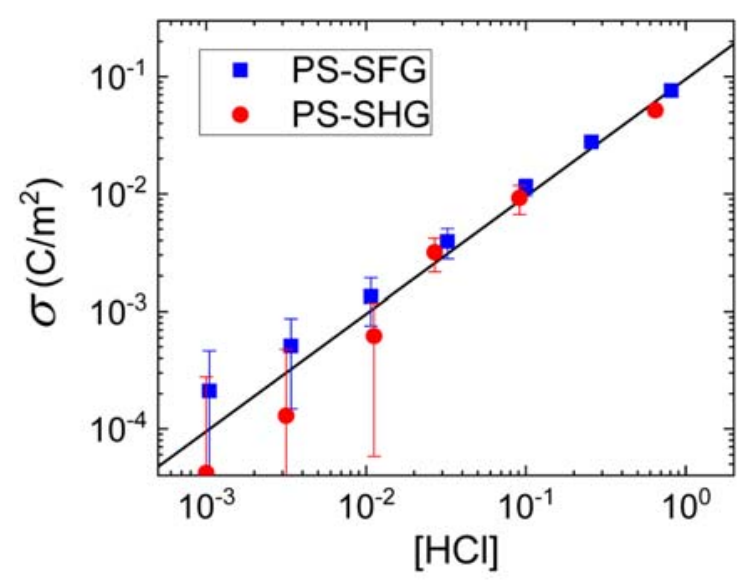

Fig. S17. Surface charge density $\sigma$ of the water/vapor interface measured from PS-SHG and PSSFVS for different $\mathrm{HCl}$ concentrations in water. We follow Ref. ${ }^{3}$ to estimate $\sigma$ by nonresonant phase-sensitive second harmonic generation (PS-SHG). Our PS-SHG setup was reported previously. ${ }^{3}$ The fundamental inputs for SHG were 50 -fs pulses at $1270 \mathrm{~nm}$ with $1 \mathrm{kHz}$ repetition rate, and the measurement was conducted with $\mathrm{S}$-in/P-out configuration at room temperature. Similar to SFG, complex $\chi_{S, \text { eff }}^{(2)}$ obtained from the PS-SHG spectroscopy can be expressed by Eq. (1) in the main text. We, again, obtain $\left[\chi_{S, e f f}^{(2)}\right]_{\sigma=0}=\chi_{B I L, 0}^{(2)}$ from the neat water surface, and approximate $\left[\chi_{B I L}^{(2)}\right]_{\sigma} \approx \chi_{B I L, 0}^{(2)}$. We then follow Eq. (1) in the main text to deduce $\chi_{E D L}^{(2)}$ for different $\mathrm{HCl}$ concentrations from the difference between the measured $\chi_{S, \text { eff }}^{(2)}$ and $\chi_{B I L, 0}^{(2)}$, and further deduce $\sigma$ from $\chi_{E D L}^{(2)}$ through the GC theory with the $\chi_{B}^{(3)}$ for SHG characterized earlier ${ }^{3}$. It is seen that $\sigma$ deduced from PS-SHG is in agreement with that from PS-SFVS for [HCl] $=10^{-2} \sim 0.8 \mathrm{M}$, supporting that the approximations adopted in PS-SFVS and PS-SHG analyses are likely valid. 


\section{Section S7. Effect of discrete surface charges on the GC theory}

We follow Ref. ${ }^{9}$ to discuss the effect of surface charge discretization on the EDL and its implications for our data analysis. The motivation is to examine whether the GC theory that ignores discrete nature of the surface charges is valid or not when the densities of bulk ions and surface adsorbed ions are very dilute (molar faction of ions $<<1$ ) in this presented study.

Different from a uniformly charged interface assumed in the GC theory, the discrete surface charges may cause a complicated electric field distribution, $E_{0}(x, y, z)$, that varies in both the $x-y$ plane and the surface normal $z$. Here we consider the dilute limit, where the mean distance between neighboring discrete surface charges, $a_{d}$, is much longer than the size of hydrated ions and the Bjerrum length. We can thus approximate the surface charges as point charges. Distribution of $E_{0}(x, y, z)$ is then dictated by the Debye screening length $\lambda_{D}$ with respect to the scale of $a_{d}$. Madurga et. al. has used Monte Carlo simulations to study the discretely charged interface of 1:1 electrolyte solutions with $\lambda_{D} / a_{d}=0.15 \sim 3.65,{ }^{9}$ and concluded that the depth-dependent ion density in the EDL, associated with the averaged $E_{0}(x, y, z)$ over the $x-y$ plane, shows no difference with the prediction of the GC theory under the point-charge approximation. Experimentally, we found $K\left(\mathrm{H}_{3} \mathrm{O}^{+}\right) \sim 11.2 \AA$ from the hydrogen-halide solutions below $0.3 \mathrm{M}$, corresponding to $\lambda_{D} / a_{d}=$ 0.25. (Note that $a_{d}\left[\approx \rho\left(\mathrm{H}_{3} \mathrm{O}^{+}\right)^{-1 / 2}\right]$ and $\lambda_{D}$ are both proportional to $\left[\mathrm{H}^{+}\right]^{-1 / 2}\left(=\left[\mathrm{X}^{-}\right]^{-1 / 2}\right)$ so that their ratio is independent to the bulk ion concentration.) It is noticed that the experimental value of $\lambda_{D} / a_{d}$ is within the range of Madurga's simulations, supporting validity of the GC theory in calculating $E_{0}(z)$ as the averaged electric field on the $x-y$ plane in our study.

The above discussion is also reminiscent of the earlier PS-SFVS study of the fatty acid monolayer/water interface, ${ }^{1}$ in which the $\mathrm{pH}$-dependent $\mathrm{SFG}$ spectral change was analyzed by the $\mathrm{GC}$ theory with the literature value of $\mathrm{p} K \mathrm{a}$ quantitatively well. For such an interface at bulk $\mathrm{pH} \sim 6$, 
the fatty acid monolayer is weakly ionized with $\sigma /|e| \sim 7 \times 10^{-3} \mathrm{~nm}^{-2},{ }^{1}$ which is readily near the detection limit of the presented study (Fig. 3A in the main text). It implies that the GC theory is feasible to calculate $E_{0}(z)$ near the weakly charged interfaces in this study, at least, for $\sigma$ above our detection limit.

\section{Section S8. Effect of the adsorbed ions on the BIL spectrum}

We have adopted the $\operatorname{Im} \chi_{S, \text { eff }}^{(2)}\left(\omega_{I R}\right)$ spectra in the $3600-3750 \mathrm{~cm}^{-1}$ region to examine the ion-induced structural change of the BIL, and found that the consequent $\Delta \chi_{B I L}^{(2)}\left(\omega_{I R}\right)$ is not important for $[\mathrm{HCl}]<0.8 \mathrm{M},[\mathrm{HBr}]<0.8 \mathrm{M}$, or $[\mathrm{HI}]<0.2 \mathrm{M}$ (see Fig. $1 \mathrm{~B}$ and $1 \mathrm{C}$ in the main text and SI Fig. S15 for details). Further examinations are given here for checking consistency of this argument in relation to other experimental/theoretical signatures.

1. It is expected that the ion-induced structural change of the BIL is hardly detected when the surface coverage of the adsorbed ions is very low. If the partitioning coefficients of ions $K$ deduced from the low-concentration SFG data (with the approximations justified) remains valid for high ion concentrations, we could estimate the maximum surface ion coverage in our experiments without noticeable $\Delta \chi_{B I L}^{(2)}\left(\omega_{I R}\right)$ in the $3600-3750 \mathrm{~cm}^{-1}$ region (see Fig. S15), which is $4.9 \%, 0.04 \%, 1.3 \%$, and $0.8 \%$ of the monolayer for $\mathrm{H}_{3} \mathrm{O}^{+}, \mathrm{Cl}^{-}, \mathrm{Br}^{-}$, and $\mathrm{I}^{-}$ions, respectively. These values of the surface ion coverage are reasonably small for explaining the undetectable $\Delta \chi_{B I L}^{(2)}\left(\omega_{I R}\right)$. However, we cannot rule out the possibility that the partitioning coefficient may differ when bulk ion concentration is high. 
2. Gaigeot et. al. used ab initio MD simulation to calculate $\chi_{B I L}^{(2)}\left(\omega_{I R}\right)$ and $\chi_{E D L}^{(2)}\left(\omega_{I R}\right)$ spectra separately for the acidic-solution/vapor interface. ${ }^{10}$ Results show that the $\chi_{B I L}^{(2)}\left(\omega_{I R}\right)$ spectrum is invariant when hydronium ions with a surface coverage of $\sim 2.5 \%$ of monolayer emerge at the water/vapor interface. It is in agreement with our above quantitative examination.

3. Again, the consistency of $\sigma$ deduced from the PS-SHG and PS-SFVS for the HClsolution/vapor interface (Fig. S17) supports the validity of the approximation, $\left[\chi_{B I L}^{(2)}\left(\omega_{I R}\right)\right]_{\sigma} \cong$ $\chi_{B I L, 0}^{(2)}\left(\omega_{I R}\right)$, for $[\mathrm{HCl}]$ up to $0.8 \mathrm{M}$.

Section S9. $\chi_{B}^{(3)}\left(\omega_{I R}\right)$ spectrum of bulk water

$\chi_{B}^{(3)}\left(\omega_{I R}\right)$ spectrum reported in Ref. ${ }^{1}$ was adopted in our spectral analysis. Particularly, we used a two-band Lorenzian model to fit the measured complex $\chi_{B}^{(3)}\left(\omega_{I R}\right)$ in Fig. 2(b) and 4(b) in Ref. ${ }^{1}$, and the fitting curve presenting a smoothened $\chi_{B}^{(3)}\left(\omega_{I R}\right)$ spectrum was used to analyze our SFG spectra for better signal-to-noise ratio. Expression and parameterization of the two-band Lorenzian model are given by

$$
\chi_{B}^{(3)}\left(\omega_{I R}\right)=\chi_{N R}^{(3)}+\sum_{q=1,2} \frac{A_{q}}{\omega_{I R}-\omega_{q}+i \Gamma_{q}}
$$

where $\chi_{N R}^{(3)}=-1.32 \times 10^{-21} \mathrm{~m}^{2} \mathrm{~V}^{-2}, \omega_{1} /(2 \pi c)=3228 \mathrm{~cm}^{-1}, \omega_{2} /(2 \pi c)=3400 \mathrm{~cm}^{-1}, A_{1}=$ $7.28 \times 10^{-9} \mathrm{~m}^{2} \mathrm{~V}^{-2} \mathrm{sec}^{-1}, \quad A_{2}=2.51 \times 10^{-9} \mathrm{~m}^{2} \mathrm{~V}^{-2} \mathrm{sec}^{-1}, \quad \Gamma_{1} /(2 \pi c)=120 \mathrm{~cm}^{-1}$, and $\Gamma_{2} /$ $(2 \pi c)=100 \mathrm{~cm}^{-1}$, with $c$ the light speed. 


\section{Reference}

1. Wen, Y.-C.; Zha, S.; Liu, X.; Yang, S. S.; Guo, P.; Shi, G.; Fang, H.; Shen, Y. R.; Tian, C. S., Unveiling microscopic structures of charged water interfaces by surface-specific vibrational spectroscopy. Physical Review Letters 2016, 116, 016101.

2. Sun, S. M.; Liang, R. D.; Xu, X. F.; Zhu, H. Y.; Shen, Y. R.; Tian, C. S., Phase reference in phase-sensitive sum-frequency vibrational spectroscopy. Journal of Chemical Physics 2016, 144 (24), 244711.

3. Dalstein, L.; Chiang, K.-Y.; Wen, Y.-C., Direct quantification of water surface charge by phase-sensitive second harmonic spectroscopy. Journal of Physical Chemistry Letters 2019, 10,5200 .

4. Rhine, P.; Hale, G. M.; Querry, M. R., Infrared optical constants of aqueous solutions of electrolytes. Acids and bases. Journal of Physical Chemistry 1974, 78 (14), 1405-1410.

5. Leyendekkers, J. V.; Hunter, R. J., Refractive index of aqueous electrolyte solutions. Extrapolations to other temperatures, pressures, and wavelengths and to multicomponent systems. Journal of Chemical and Engineering Data 1977, 22 (4), 427-431.

6. Urashima, S. H.; Myalitsin, A.; Nihonyanagi, S.; Tahara, T., The topmost water structure at a charged silica/aqueous interface revealed by heterodyne-detected vibrational sum frequency generation spectroscopy. Journal of Physical Chemistry Letters 2018, 9 (14), 4109-4114.

7. Borukhov, I.; Andelman, D.; Orland, H., Steric effects in electrolytes: A modified PoissonBoltzmann equation. Physical Review Letters 1997, 79 (3), 435-438.

8. Kilic, M. S.; Bazant, M. Z.; Ajdari, A., Steric effects in the dynamics of electrolytes at large applied voltages. I. Double-layer charging. Physical Review E 2007, 75 (2), 021502.

9. Madurga, S.; Martin-Molina, A.; Vilaseca, E.; Mas, F.; Quesada-Perez, M., Effect of the surface charge discretization on electric double layers: A Monte Carlo simulation study. Journal of Chemical Physics 2007, 126 (23), 234703.

10. Pezzotti, S.; Gaigeot, M. P., Spectroscopic BIL-SFG invariance hides the chaotropic effect of protons at the air-water interface. Atmosphere 2018, 9 (10), 396. 